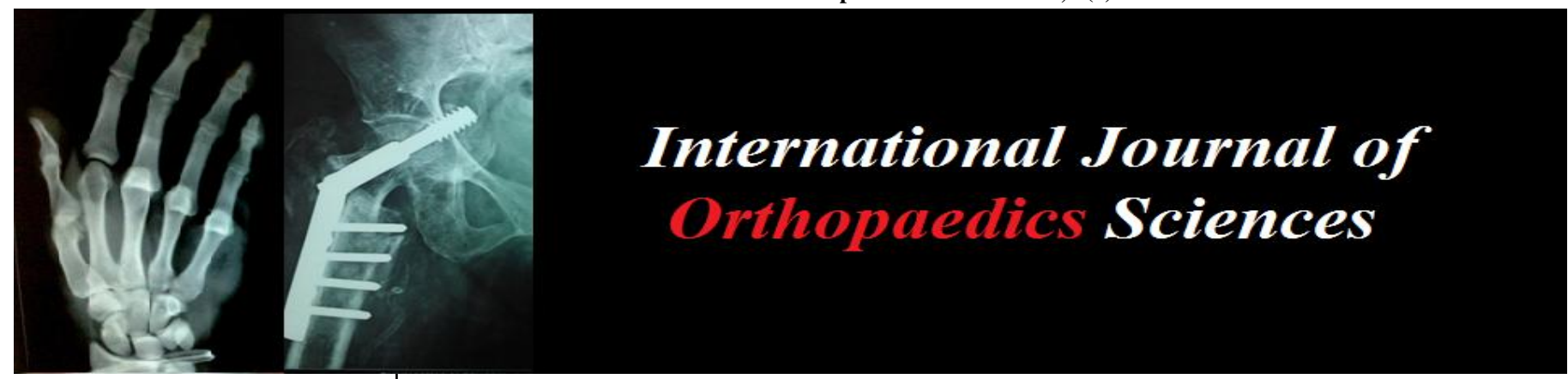

ISSN: $2395-1958$

IJOS 2017; 3(4): 612-614

(C) 2017 IJOS

www.orthopaper.com

Received: 24-08-2017

Accepted: 25-09-2017

Navneet Agarwal

R.G. Kar Medical College \&

Hospital, Kolkata, West Bengal

India

Sabyasachi Santra

College of Medicine \& Sagore

Dutta Hospital, Kolkata,

West Bengal India
Correspondence

Navneet Agarwal

R.G. Kar Medical College \&

Hospital, Kolkata, West Bengal

India

\section{Open reduction with ulnar osteotomy for chronic post- traumatic radial head dislocation in children}

\author{
Navneet Agarwal and Sabyasachi Santra
}

DOI: https://doi.org/10.22271/ortho.2017.v3.i4i.84

Abstract

Purpose: A number of surgical techniques with variable results and complications have been described for the treatment of chronic post-traumatic radial head dislocations or the missed Monteggia lesions. This study aims to evaluate open reduction of radial head with ulnar corrective osteotomy with rigid fixation with plate, without annular ligament reconstruction or repair.

Methods: 11 patients were treated between 2011 and 2016, 7 were male while 4 were female, average age being 7.8 years (range 6-11.5 years). Right side was involved in 8/11. The average interval between injury and surgery was 4 months (range 2 to 11 months). Patients were followed up for mean period of 13 months (7-21 months), assessed clinically for elbow function, stability, pain, deformity and radiologically for reduction, periarticular ossifications, signs of malformation of the radial head or osteoarthritic changes.

Results: All patient regained painless function of the elbow, adequate range of elbow and forearm motion with maintenance of the radial head reduction.

Conclusion: Ulnar osteotomy with angulation and lengthening, provides adequate aid in reduction of radial head, without requiring additional annular ligament procedures.

Keywords: Radial head, ulnar osteotomy, missed Monteggia lesions

\section{Introduction}

Dislocation of the radial head can be seen in combination with a fracture of the ulna (Monteggia lesion) or as a Monteggia equivalent lesion without fracture of the ulna. Bado's classification divides Monteggia lesions into true Monteggia lesions and 'equivalent lesions' based on the mechanism of the injury and the direction of the dislocation [1]. These injuries are rare in children and dislocation of the radial head can be overlooked easily.

Persistent radial head dislocation may cause deformity like increasing valgus deformity of the elbow with ulnar or radial neuropathy, loss of flexion due to obstruction by the radial head, or loss of rotation due to malformation of the radial head, stiffness and instability, which may lead to functional impairment but may also develop secondary degenerative arthritis lately ${ }^{[2-4]}$. The treatment of chronic radial head dislocation remains controversial and many operations for chronic dislocation of the radial head show unsatisfactory results and few are described as salvage procedures. The procedures include ulnar and radial osteotomies, open or closed reduction of the radial head, repair or reconstruction of the annular ligament, temporary fixation of the radial head with a transarticular wire, or some combination of these techniques. Complications including redislocation, stiffness, elbow instability, nonunion at the osteotomy site, avascular necrosis of the radial head, nerve injury and infection ${ }^{[5-11]}$.

This study aims to evaluate the effectiveness of open reduction of radial head with the aid of ulnar osteotomy with angulation and lengethening of ulna, without annular ligament repair or reconstruction.

\section{Materials and Methods}

11 patients were treated between 2011 and 2016 for treatment of post traumatic persistent radial head dislocation (4 weeks or later), 7 were male while 4 were females, average age being 7.8 years (6-11.5 years). Right side was involved in 8/11. Congenital radial head dislocation was ruled out with standard skiagrams of both the elbows. The average interval between injury and surgery was 4 months (range 2 to 11 months). 
As per Bado classification, 6/11 had anterior dislocation (Type I), 2/11 had lateral dislocation (Type III), 3/11 had Monteggia equivalent lesion, isolated anterior dislocation of radial head. None of the patients had a history of previous elbow pathology or surgery.

Patients were followed up for mean period of 13 months (7-21 months), assessed clinically and radiologically. Radiographs evaluated for reduction of the radial head, periarticular ossifications, and secondary signs of malformation of the radial head or osteoarthritic changes.

\section{Surgical technique}

In supine position, with prophylactic antibiotic and tourniquet control, Kocher approach was used, extending the incision along ulnar shaft to expose both the radio-capitellar joint and ulna shaft. Fibrous scar tissue around radial head was excised and reduction of radial head attempted after performing an oblique ulnar osteotomy, either at the site of ulnar malunion or at proximal ulnar metaphysis. Stability of radial head reduction was checked and confirmed under image intensifier, then osteotomy site was fixed with small DCP in neutral mode or Recon plate after proper contouring. Stability of radial head reduction was again assessed. No annular ligament repair or reconstruction was attempted. In one case, where radial head stability was in doubt for mild anterior subluxation, additional radio-capitellar $\mathrm{K}$ wire fixation was done after adequate reduction, which was removed at 3 weeks, for the fear of pin breakage. Plaster immobilisation was maintained in all for 6 weeks post-operatively, with elbow in $90^{\circ}$ flexion and forearm in neutral position. Active physiotherapy was advised after 6 weeks post-operatively.

The mean angulation at ulnar osteotomy site was $16.2^{\circ}$ (range $10^{\circ}-30^{\circ}$ ), and the mean distraction was 6.9 (range 4-15) $\mathrm{mm}$.

\section{Results}

No pre-operative or post-operative ulnar or radial neuropathy was noted. Radial head reduction was adequate in $11 / 11$ patients. 6/11 had pain pre-operatively which was relieved post-operatively. On stress testing in coronal plane, stability was found comparable to normal side. All were pain free at final follow-up.

Mean increase in flexion was $20^{\circ}$ (range $10^{\circ}-40^{\circ}$ ). There was reduction in supination in 2 patients by $20^{\circ}$, while supination remained same post-operatively in 7 , improved by $30^{\circ}$ in $2 / 11$.

The radiographs at follow-up showed no degenerative changes or periarticular ossifications. Radial head alignment was acceptable.

Adequate union was achieved in all cases at the ulna osteotomy site.

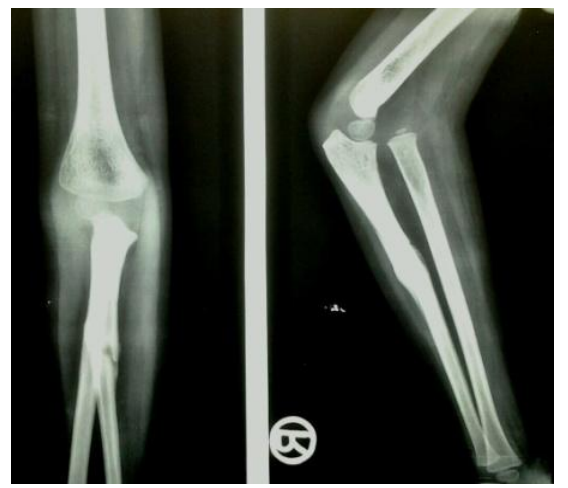

Fig 1: Skiagram showing maluniting Bado type I Monteggia fracture dislocation

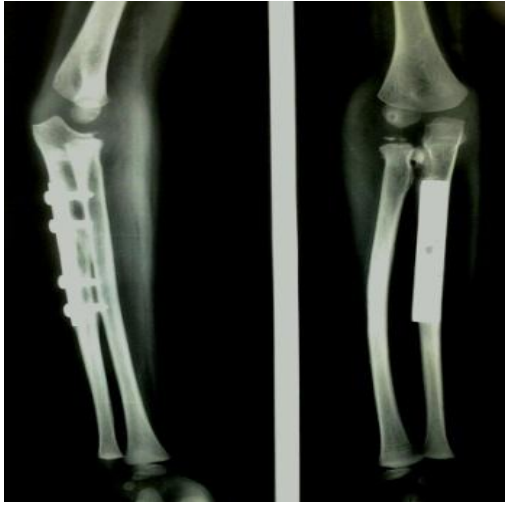

Fig 2: Skiagram of the same patient at 6 months follow-up

\section{Discussion}

The radial head plays a key role in maintaining stability of the elbow joint. Unreduced dislocation of the radial head for more than 4 weeks is considered to be chronic. Although there is no argument against reducing a dislocated radial head immediately after trauma, there are arguments against reducing a chronic dislocation ${ }^{[12]}$.

In unreduced radial head, the radio-capitellar articulation will progressively undergo dysplastic changes due to the lack of joint restraint, leading to long-term consequences. In reconstructive surgery for chronic dislocation of the radial head, sequelae such as loss of elbow function, redislocations and complications have been described ${ }^{[13,14]}$.

Surgery is recommended for active patients as long-standing radial head dislocation will possibly cause restriction of elbow flexion, deformity of the radial head, overgrowth of the radius, instability of the elbow joint and early osteoarthritic changes of the elbow joint. Interval between injury and treatment, the age of the patient and the amount of joint incongruity plays an important role ${ }^{[15]}$.

Hirayama et al and Stoll et al reported that reconstruction could be successfully achieved in children up to ten years of age and at least four years after the initial injury ${ }^{[11,16]}$.

Best et al recommended ligament reconstruction without osteotomy, but complications after ligament reconstruction alone comprise osteolytic changes, narrowing or growth disturbance of the radial neck, and restricted pronation and supination ${ }^{[17]}$. Moreover, the additional dissection required to reconstruct the annular ligament might result in elbow stiffness, avascular necrosis of the radial head, heterotopic ossification, or radio-ulnar synostosis ${ }^{[13,18,19]}$.

Horii et al recommended, angulation and elongation of the ulna are often necessary to maintain reduction of a chronic dislocation of the radial head ${ }^{[20]}$. Mehta recommended against internal fixation to allow subsequent ulnar remodelling. However prolonged immobilisation in a cast can cause contracture and possibly delayed union or even non-union ${ }^{[21]}$. Koslowsky et al. have described an external fixator as fixation, a technique that provides the ability to do a percutaneous osteotomy and perform the correction through a three-dimensional correction of the ulnar axis ${ }^{[22]}$.

In the series described in this study rigid fixation of the osteotomy site was done using a plate to prevent secondary displacement of the osteotomy. A disadvantage of this technique was that plate removal was necessary in all cases.

\section{Conclusion}

Open reduction of Radial head and corrective ulnar osteotomy, with lengthening and angulation with plate fixation, even without annular ligament reconstruction or 
repair, shows good results for missed radial head dislocations in children.

\section{References}

1. Bado JL. The Monteggia lesion. Clin Orthop Relat Res 1967; 50:71-86

2. Dormans JP, Rang M. The problem of Monteggia fracture-dislocations in children. Orthop Clin North Am 1990; 21:251-266

3. Chen WS. Late neuropathy in chronic dislocation of the radial head. Report of two cases. Acta Orthop Scand. 1992; 63:343-344

4. Holst-Nielsen F, Jensen V. Tardy posterior interosseous nerve palsy as a result of an unreduced radial head dislocation in Monteggia fractures: a report of two cases. J Hand Surg. 1984; 9:572-575

5. Hasler CC, Von Laer L, Hell AK. Open reduction, ulnar osteotomy and external fixation for chronic anterior dislocation of the head of the radius. J Bone Joint Surg Br 2005; 87:88-94.

6. Rodgers WB, Waters PM, Hall JE. Chronic Monteggia lesions in children. Complications and results of reconstruction. J Bone Joint Surg Am. 1996; 78:13221329

7. Futami T, Tsukamoto Y, Fujita T. Rotation osteotomy for dislocation of the radial head. 6 cases followed for 7 (310) years. Acta Orthop Scand. 1992; 63:455-456.

8. Hui JH, Sulaiman AR, Lee HC, Lam KS, Lee EH. Open reduction and annular ligament reconstruction with fascia of the forearm in chronic monteggia lesions in children. $\mathbf{J}$ Pediatr Orthop. 2005; 25:501-506.

9. Devnani AS. Missed Monteggia fracture dislocation in children. Injury. 1997; 28:131-133.

10. Hurst LC, Dubrow EN. Surgical treatment of symptomatic chronic radial head dislocation: a neglected Monteggia fracture. J Pediatr Orthop. 1983; 3:227-230.

11. Hirayama T, Takemitsu Y, Yagihara K, Mikita A. Operation for chronic dislocation of the radial head in children. Reduction by osteotomy of the ulna. J Bone Joint Surg Br. 1987; 69:639-642.

12. Morrey BF, Tanaka S, An KN. Valgus stability of the elbow. A definition of primary and secondary constraints. Clin Orthop Relat Res. 1991; 265:187-195.

13. Oner FC, Diepstraten AF. Treatment of chronic posttraumatic dislocation of the radial head in children. $\mathrm{J}$ Bone Joint Surg Br. 1993; 75:577-581.

14. Ring D, Waters PM. Operative fixation of Monteggia fractures in children. J Bone Joint Surg Br. 1996; 78:734739

15. Kim HT, Conjares JNV, Suh JT, Yoo IC. Chronic head dislocation in Children, Part 1: Pathologic changes preventing stable reduction and surgical correction. J Pediatr Orthop. 2001; 22:583-590.

16. Stoll TM, Willis, Paterson DC. Treatment of the missed Monteggia fracture in the child. J Bone Joint Surg Br 1992; 74:436-440.

17. Best TN. Management of old unreduced Monteggia fracture dislocations of the elbow in children. J Pediatr Orthop. 1994; 14:193-199.

18. Gyr BM, Stevens PM, Smith JT. Chronic Monteggia fractures in children: outcome after treatment with the Bell- Tawse procedure. J Pediatr Orthop Br. 2004; 13:402-406.

19. De Boeck H. Radial neck osteolysis after annular ligament reconstruction. A case report. Clin Orthop Relat
Res 1997, 94-98.

20. Horii E, Nakamura R, Koh S. Surgical treatment for chronic radial head dislocation. J Bone Surg Am. 2002; 84:1183-1188

21. Mehta SD. Flexion osteotomy of ulna for untreated Monteggia fracture in children. Ind J Surg. 1985; 47:1519.

22. Koslowsky TC, Mader K, Wulke AP. Operative treatment of chronic Monteggia lesion in younger children: a report of three cases. J Shoulder Elbow Surg 2006; 15:119-121. 